\title{
Proximal Phalanx Fracture Management
}

\author{
Tim T. Lögters', Hannah H. Lee ${ }^{2}$, Sebastian Gehrmann', \\ Joachim Windolf', and Robert A. Kaufmann²
}

\begin{abstract}
Background: The goal of proximal phalangeal fracture management is to allow for fracture healing to occur in acceptable alignment while maintaining gliding motion of the extensor and flexor tendons. Methods: We reviewed the most current literature on various treatment methods of proximal phalanx fractures, focusing on the indications and outcomes of nonoperative as well as operative interventions. Results: Stable fractures can be successfully treated nonoperatively, whereas unstable injuries benefit from surgery. Regardless of the surgical intervention employed, the overriding goal is to restore anatomy and impart enough stability to allow for early motion. The surgical dissection contributes to soft tissue scarring and should be minimized. Conclusions: Clinical success is achieved when acceptable fracture alignment and stability occur in the setting of unobstructed tendon gliding and early active range of motion.
\end{abstract}

Keywords: phalanx, fracture, plate, stability, K-wire, screw

\section{Fracture Stability Assessment}

\section{Deforming Forces}

Biomechanical studies have shown that the proximal phalanx experiences forces approaching 25 Newton during common activities, such as jar opening. ${ }^{13}$ The interosseous muscles insert onto the base of the proximal phalanx and flex the proximal fracture fragment, leading to an apex volar deformity. ${ }^{23}$ The flexor and extensor tendons impart a longitudinal compression force that shortens the phalanx and extends the distal fragment.

\section{Stable Fracture}

A phalanx fracture is stable if deforming forces do not affect its alignment during early mobilization of adjacent joints. ${ }^{34}$ The ability to withstand deforming forces is influenced by the fracture pattern and degree of displacement, with the most stable being a nondisplaced transverse fracture. ${ }^{22,34}$ Nondisplaced fractures often exhibit an intact periosteum, which imparts further stability.

\section{Unstable Fracture}

An unstable fracture is one where otherwise insignificant forces would likely cause displacement or where a closed reduction has been unsuccessful. In this situation, additional stability imparted by surgical fixation is required. $8,22,34$ Spiral, long oblique, and comminuted fractures fall into this category. A greater degree of initial displacement, need for fracture reduction, and the displacement of fragments increase the likelihood of instability. Assessing stability may be difficult using radiographs alone.

\section{Nonoperative Management}

Nonoperative treatment is recommended for stable proximal phalanx fractures. Immobilization in an "intrinsic-plus" position through metacarpophalangeal (MCP) joint flexion reduces the displacing force of the interossei and also shifts the extensor tendon distally so that two-thirds of the proximal phalanx is embraced by the extensor mechanism, adding to the overall fracture stabilization. ${ }^{8,14,17,22,29}$ Allowing active proximal interphalangeal (PIP) joint flexion further compresses the fracture and may be considered for particularly stable fractures.

Many stable phalanx fractures can be treated nonoperatively through close monitoring until clinical healing is noted. ${ }^{12}$ Proximal phalanx fractures will often be clinically healed 4 weeks status post injury, at which time it is unlikely

\footnotetext{
'University Clinic Düsseldorf, Germany

${ }^{2}$ University of Pittsburgh Medical Center, PA, USA
}

Corresponding Author:

Robert A. Kaufmann, Department of Orthopedic Surgery, University of Pittsburgh Medical Center, Suite 1010 Kaufmann Building, 347I Fifth Avenue, Pittsburgh, PA 15213, USA.

Email: kaufra@upmc.edu 
that the fracture will displace. ${ }^{8}$ A method of qualifying this is lack of discomfort when direct manual pressure is applied to the injured bone.

A prospective cohort study treated both stable (39\%) and unstable (61\%) extra-articular proximal phalanx fractures nonoperatively. ${ }^{17}$ All fractures were reduced and maintained in a dorsal MCP flexed splint. The injured finger was buddy-taped to the adjacent digit to control for rotation. The splint was removed at 3 weeks. Ninety-one percent maintained an acceptable position with an average shortening of $1.1 \mathrm{~mm}$ that resulted in a mild extensor lag.

Nonoperative treatment using a hand-based (wrist free) thermoplastic splint demonstrated no tendon adhesion, contracture, infection, nonunion, or malunion. ${ }^{29}$ Seventy-two percent attained near normal total active motion (TAM), and only $2(6 \%)$ had fair to poor results.

A prospective randomized multicenter study was carried out to compare outcome differences with the wrist immobilized or left free. ${ }^{14}$ No significant differences were noted in fracture angulation, finger motion, or PIP extensor lag. Wrist motion was significantly greater for the wrist free group at 6 weeks, but this difference disappeared at 12 weeks.

\section{Operative Management}

The ultimate goal of achieving fixation is to create a biomechanical environment capable of withstanding gentle forces that are associated with early range of motion. Ideally, the fixation itself would not impart significant friction to the extensor or flexor mechanism.

\section{Soft Tissue Management}

A thin soft tissue envelope surrounds the proximal phalanx and must be handled delicately with some advocating elevating the periosteum with the extensor tendon. ${ }^{18}$ Indirect reduction methods, where fragments are reduced with fluoroscopic and not direct visualization, may be beneficial. Retracting the extensor mechanism instead of surgically splitting it optimizes extensor mechanism function and minimizes scarring. ${ }^{26}$ Fixation under the extensor tendon is to be avoided; if unavoidable, screws should be countersunk to minimize friction. ${ }^{18,27}$ Radial or ulnar midaxial positions beginning distal to the lateral bands and ending proximal to the collateral ligaments of the PIP joint represent an ideal location to avoid friction between the hardware and the mobile extensor tendon. ${ }^{26,27}$ If need be, 2 plates can be placed on both radial or ulnar sides and the lateral bands can be partially excised to facilitate more proximal plate placement.

\section{Healing Methods: Indirect}

Nonoperative treatment will always create indirect fracture healing, whereas operative intervention will encourage either direct or indirect healing based on the degree of stability that is imparted through the surgical effort. ${ }^{31}$ Indirect bone healing is primarily associated with Kirschner wire (K-wire) fixation techniques. The radiographic appearance often lags behind clinical healing with fracture gaps remaining visible for 4 months. Indirect healing produces excellent clinical results as long as early range of motion is ensured.

\section{K-Wire Fixation}

The goal of K-wire fixation is to create a biomechanical environment that is stable enough to allow early postoperative mobilization. ${ }^{3}$ Relative stability encourages abundant callus formation. ${ }^{31,32}$ A diameter of at least $0.9 \mathrm{~mm}$ is recommended for the proximal phalanx. ${ }^{22}$ Understanding various methods of pin placement is important as adequate stability may require multiple $\mathrm{K}$-wires be placed in different orientations. ${ }^{4,22}$ Four crossed K-wires obtain the highest rigidity in a transverse fracture pattern. In oblique fractures, bending, torsion, and distraction forces are best neutralized by wires placed perpendicular to the fracture. Compression loading is best resisted by $\mathrm{K}$-wires placed in line with the shaft.

$\mathrm{K}$-wires are particularly valuable in the management of proximal third fractures, where the extensor tendon cloaks this region and where permanent plates may cause adhesions. ${ }^{11}$ The 2 primary techniques for K-wire insertion in this area are transarticular and periarticular (Figure 1).

The transarticular technique crosses the MCP joint, ${ }^{3}$ whereas the periarticular technique places the pins from radial and ulnar starting points on the base of the proximal phalanx. $^{10,11}$

A cadaver study showed that K-wires placed away from the extensor tendon create less of a tether to the PIP joint than those placed through or adjacent to it. ${ }^{32} \mathrm{~A}$ transarticular $\mathrm{K}$-wire insertion through the extensor tendon led to a $34^{\circ}$ loss of PIP joint flexion, whereas periarticular insertion at the midaxial line led to only a $12^{\circ} \mathrm{PIP}$ joint flexion restriction.

Proximal phalanx fractures treated with 2 parallel transarticular K-wires inserted radial and ulnar to the extrinsic extensor tendon showed good overall TAM of $265^{\circ}$ at final follow-up and a mean range of PIP joint motion of $96^{\circ} .{ }^{19}$

The periarticular pinning technique for extra-articular proximal phalanx fractures was reviewed with $63 \%$ achieving TAM of $260-270^{\circ} .{ }^{10}$ Seven percent developed stiffness severe enough to warrant tenolysis.

One comparison study between transarticular and periarticular pinning methods showed neither fixation method to be superior. ${ }^{11}$ The MCP was not stiffer in the transarticular group despite 3 weeks of K-wire fixation and yet there was a trend for an overall higher complication rate in this group. Another comparison study, however, reviewed these two methods in 40 fractures and reported that the periarticular group demonstrated significantly better outcomes. ${ }^{1}$ 


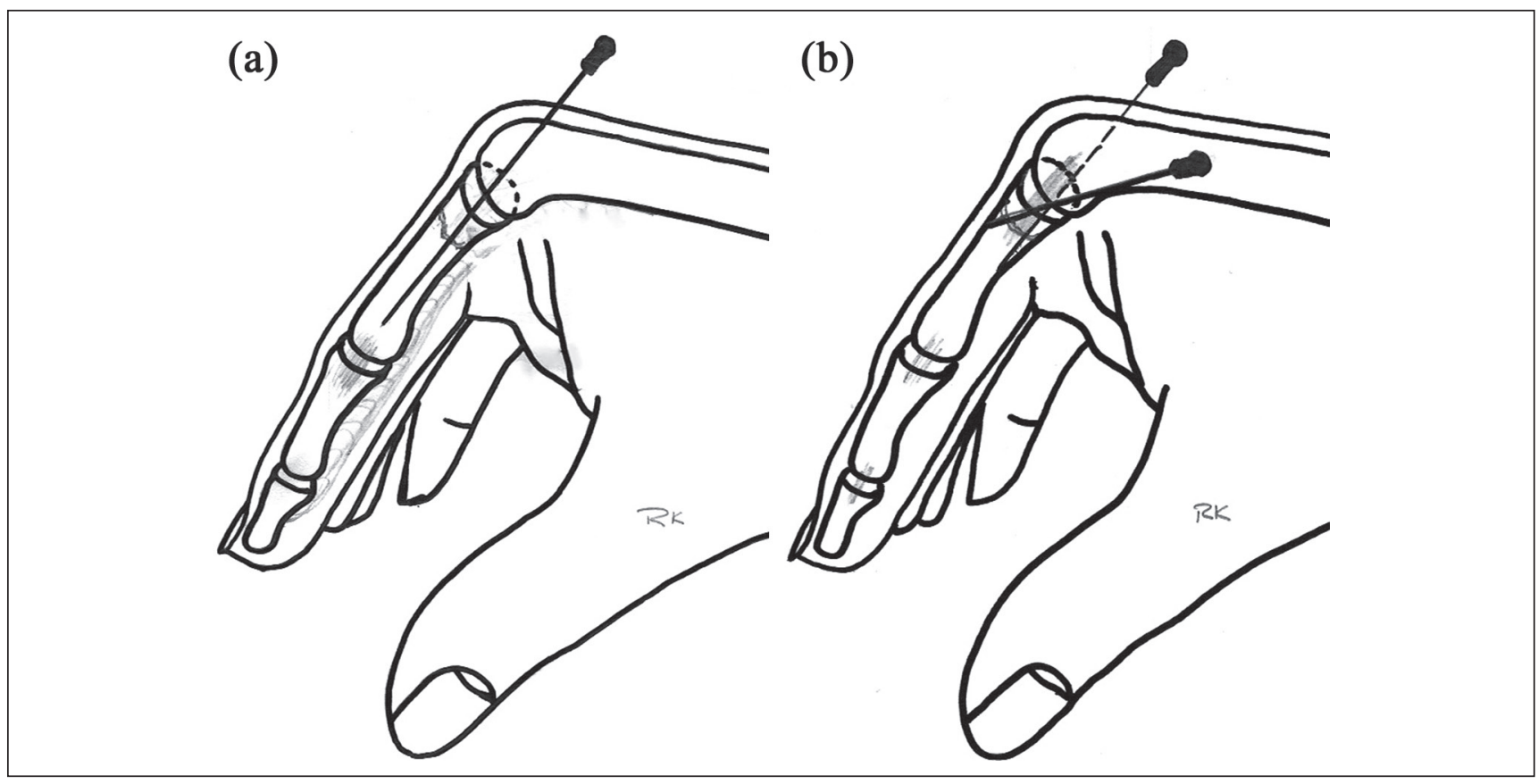

Figure I. Kirschner wire (K-wire) fixation methods for proximal phalanx fracture. (a) Transarticular technique where the K-wire crosses the metacarpophalangeal joint prior to crossing the fracture site. (b) Periarticular technique where the pins start radial and ulnar from the base of the proximal phalanx and cross the fracture site.

\section{Healing Methods: Direct}

Rigid fixation with lag screws, plates, or both leads to a stable healing environment, in which direct bone healing is to be expected. ${ }^{31}$ Direct fracture healing minimizes callus formation and thus obviates the potential detrimental effect that callus may have on tendon gliding. This healing type is only advantageous when it occurs in association with fixation that does not itself adversely affect tendon motion.

Unlike K-wires which are removed at 3 to 4 weeks, screws and plates are intended to remain in place and may directly result in finger stiffness related to adhesions between the hardware and the extensor mechanism. ${ }^{21}$ Therefore, any hardware that is unnecessarily large or has sharp edges, such as prominent screw heads or a plate, will inevitably lead to mechanical irritation and increased inflammation. ${ }^{25}$

\section{Screw Fixation}

Indications for screw fixation are long oblique and spiral fractures. ${ }^{8,22}$ They are best placed as lag screws that compress 2 fractured fragments and are ideally countersunk so as not to irritate the extensor tendons. This fracture compression creates absolute stability and promotes direct fracture healing.

Although 2 lag screws may be adequate, ${ }^{35}$ a third screw placed in a different orientation can improve construct stability. ${ }^{8,22,34}$ Screws can also be percutaneously placed after a closed reduction effort has been performed. This latter method decreases soft tissue dissection; the benefit of which may be offset by the difficulties imparted by not directly visualizing the fracture reduction.

The results of screw and K-wire fixation of oblique and spiral proximal phalanx fractures were compared in a randomized clinical trial demonstrating no difference in the rates of malunion, range of motion, or grip strength. ${ }^{20}$ All fractures healed - in the K-wire group by indirect and in the screw group by direct healing. The study did not show either method to be superior.

\section{Intramedullary Screw Fixation}

Intramedullary cannulated headless compression screws have also been advocated for the treatment of proximal phalanx fractures. ${ }^{6,9,15}$ This technique has the advantage of avoiding periosteal stripping and allowing for almost immediate motion. Not having any metal on the bone surface minimizes adhesions. Both primary healing with minimal callus and secondary healing with prominent callus formation have been observed. ${ }^{9}$

A cadaver study assessed the amount of MCP joint cartilage damage when 2 different methods of screw placement were used (Figure 2). ${ }^{6}$ One technique places the guidewire through the MCP joint but not through the metacarpal head (intra-articular), whereas the other method places the guidewire through the metacarpal head first and then into the proximal phalanx (transarticular). After guidewire insertion, 

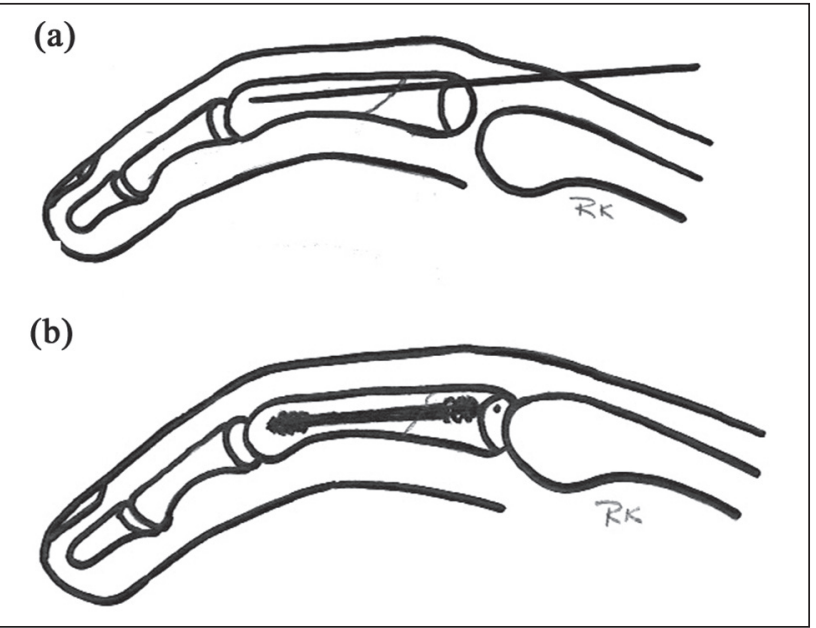

Figure 2. Intra-articular technique of intramedullary compression screw fixation of proximal phalanx fracture. (a) The guidewire is placed through the metacarpophalangeal joint but not through the metacarpal head. (b) After guidewire insertion, a cannulated headless compression screw is driven over the guidewire until the screw head is buried.

a cannulated headless compression screw is driven over the guidewire until the screw head is buried. Not surprisingly, the defect in the proximal articular surface of the proximal phalanx was not significantly different and yet a sizable cartilage defect remained in the metacarpal head when the screw had to move through it on its way into the proximal phalanx. The authors concluded that the intra-articular technique is better as it produces less cartilage damage.

Twenty-four proximal phalanx fractures treated with antegrade intramedullary cannulated headless compression screws were retrospectively reviewed. ${ }^{15}$ Transarticular technique was favored for very proximal fractures and good outcomes were reported, except one long oblique fracture that displaced after 9 days and another screw that protruded into the MCP joint.

A retrograde technique that maximally flexed the PIP joint and split the central band to allow placement of cannulated headless screws in 19 proximal phalanx fractures was reviewed. ${ }^{9}$ The technique was most effective for transverse and short oblique fractures. Two of 19 patients had a substantial PIP extensor lag. Between $13 \%$ and $18 \%$ of the articular surface at the proximal phalangeal head was damaged by the 2.5 -mm screw and $19 \%$ and $25 \%$ by the $3.0-\mathrm{mm}$ screw.

\section{Plate and Screws}

Plate fixation has become more popular and can impart greater construct rigidity when compared with other methods of fracture fixation. ${ }^{2,23,28}$ Adhesions may occur between the tendon and implant ${ }^{7,23,30}$ and are not due only to mechanical friction but also secondary to a foreign body soft tissue response. ${ }^{21,25}$ The implant material may not play a large role in the formation of stiffness as both stainless steel and titanium implants have been associated with increased inflammation. ${ }^{5,16}$ Precontoured plates have a potential benefit of reducing the risk of tendon irritation. ${ }^{33}$

Screw fixation alone was compared with screw fixation with plating in 22 phalangeal fractures that were approached with a dorsal, extensor tendon splitting incision. ${ }^{2}$ Total active motion measured at the last follow-up was significantly better for the phalangeal fractures treated with screws only, prompting the authors to discourage the use of plates and screws for spiral and oblique fractures.

Titanium plates placed through a dorsal approach with longitudinal splitting of the central slip were used to treat extra-articular proximal phalangeal fractures. ${ }^{2}$ An extensor lag was found in $67 \%$ of the fractures with many plates needing to be removed postoperatively. The authors concluded that low-profile plates placed dorsally encountered frequent adhesion problems and that postoperative finger motion was often poor. They recommended screw fixation without plating if open reduction is required.

\section{Plate and Locking Screws}

Low-profile plates that employ locking screw fixation have been introduced with the hope that they may result in less tendon adherence, but this has not been conclusively shown. ${ }^{7,23,28}$ Thinner plates are also associated with reduced strength, ${ }^{22}$ though the addition of locking screws is believed to decrease its likelihood of failure given that this construct forces catastrophic failure where all screws must fail together. Locking screws may obviate the need for placing the plate on the tension side of the injured bone. ${ }^{31}$

Highly unstable fractures with bony defects would potentially benefit from the use of locking screws. In these situations or when comorbidities increase the risk for delayed healing, locking screw fixation may enhance stability.

Plate and screw fixation improves the construct stability, but some plating systems have been shown to have inferior rigidity than 2 to 4 crossed $\mathrm{K}$-wire fixation techniques in biomechanical studies with apex volar testing. ${ }^{24} \mathrm{~A}$ biomechanical study assessed quantitative differences in the stability obtained with 5 commonly used types of internal fixation. ${ }^{4}$ Lag screws alone or in combination with a plate provided more rigidity than tension band or K-wire fixation methods, yet maximum bending moments to failure were similar between all fixation techniques leading the authors to conclude that all methods enabled early postoperative motion.

\section{Plate Location}

Plate placement on the dorsum of the proximal phalanx has been advocated historically. While this location allows for ideal fracture visualization and affords convenient plate 


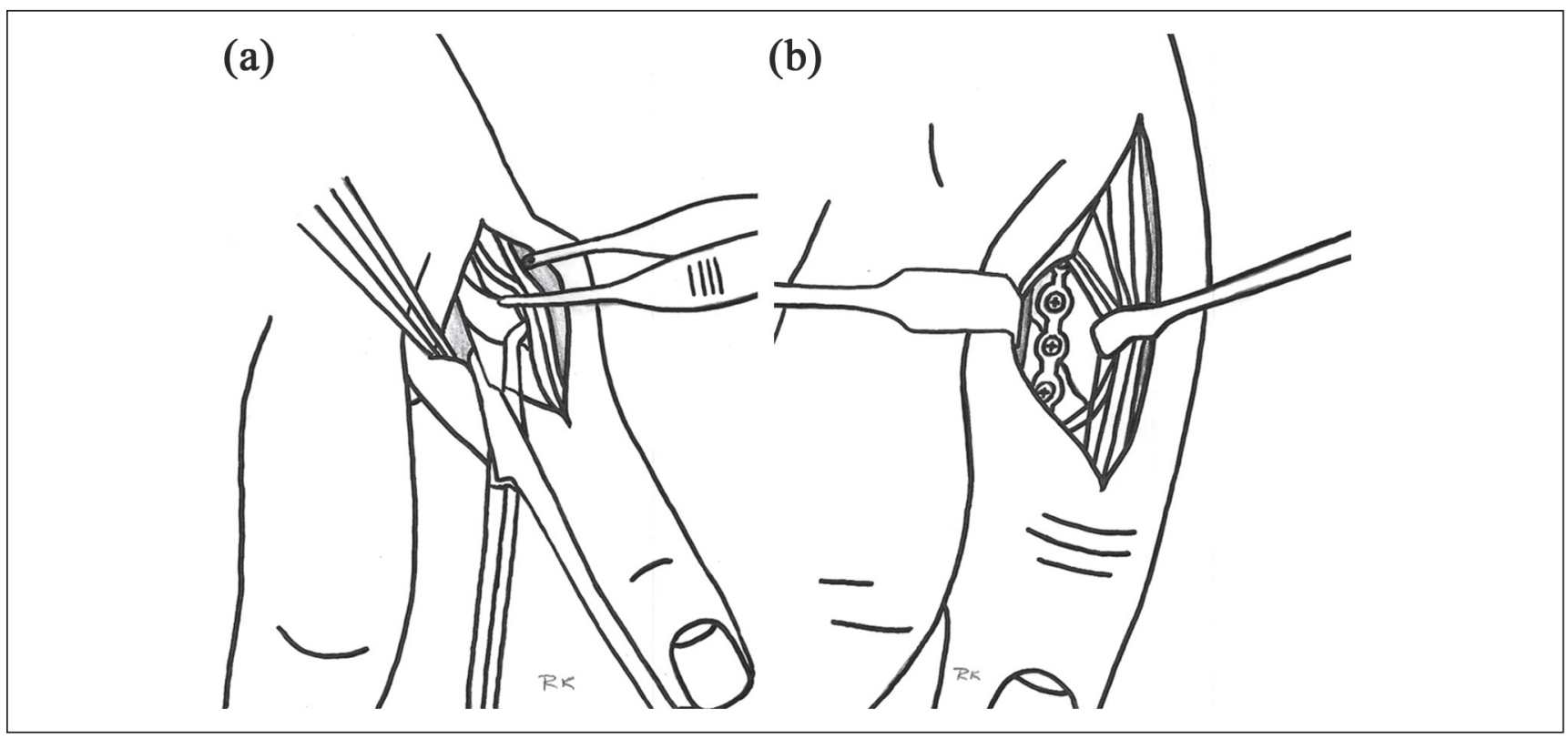

Figure 3. Radial or ulnar midaxial plate placement for proximal phalanx fractures. (a) Extensor mechanism is retracted to gain fracture visualization, instead of being split iatrogenically. (b) Radial or ulnar midaxial placement of the plate avoids tendon being in direct contact and subsequent adherence to the plate.

positioning on a flat dorsal surface, it also maximizes extensor tendon problems. ${ }^{27} \mathrm{~A}$ longitudinal splitting of the extensor mechanism may lead to an extensor lag status post repair, and the mechanical friction imparted by the plate is substantial.

For that reason, a radial or ulnar midaxial plate position carries great advantages. ${ }^{22}$ The extensor mechanism is not iatrogenically injured during exposure, and it is instead retracted to gain fracture visualization. In addition, the plates do not contribute to tendon adherence problems (Figure 3). Although technically more difficult, placing plates on the radial and ulnar sides where the extensor tendon is not in direct contact is recommended.

A cadaver biomechanical study showed that dorsal or lateral placement of stainless steel plates provided essentially identical stability. ${ }^{27}$ The authors conclude that for fractures that require plate fixation, similar mechanical stability can be achieved with either lateral or dorsal plate placement. However, when appropriate, lateral placement is encouraged as it is less invasive and helps preserve the integrity of the soft tissues.

Shimizu et al reviewed titanium plate fixation of 34 comminuted periarticular proximal phalanx fractures and concluded that lateral plating was preferred to avoid interference of the implant with extensor tendon gliding. ${ }^{33}$ However, dorsal plating was used for severely comminuted fractures. Patient age and associated soft tissue injury were primary contributors to increased finger stiffness via multivariate linear regression analysis. Although lateral versus dorsal plating did not affect degree of stiffness, the authors endorsed lateral plating as the extensor tendons were observed to be a specific cause of adhesion when a tenolysis and plate removal were performed.

Seventy-five unstable proximal phalanx fractures were treated with titanium plates and screws in 59 fractures and with screws only in 16 fractures. ${ }^{26}$ Fixation was dorsal in 33 fractures and lateral in 42 fractures. Multivariate logistic model analysis of TAM showed increased finger stiffness was associated with plate and screw fixation versus screw only fixation, as well as with dorsal versus lateral plate placement.

Another study, however, showed no difference in outcome with dorsal or lateral plate fixation. ${ }^{30}$ Final TAM was not statistically different between the 2 groups, and the complication rate was quite high, with $48 \%$ experiencing major complications.

An adhesion barrier between the plate and the soft tissues has been postulated to reduce friction when using a dorsal plate. ${ }^{21}$ A double-blinded randomized controlled trial showed mean motion and Disabilities of the Arm, Shoulder, and Hand values to be slightly better for the barrier group at 6 weeks, although not statistically significant, and this trend disappeared by 6 months.

\section{Complications}

$\mathrm{K}$-wire fixation is associated with finger stiffness, tendon adhesions, and pin site infections. ${ }^{10,11,19}$ Transarticular pinning trended toward greater complications compared with periarticular methods. ${ }^{11}$ Complications associated with intramedullary screws are cartilage damage and fracture 
displacement occurring during screw insertion. ${ }^{15}$ Plate and screw fixation is associated with stiffness, extensor lag, infection, nonunion, malunion, and hardware failure. ${ }^{23,30}$ Plates and screws may interfere with tendon gliding requiring hardware removal and tenolysis efforts. ${ }^{26,30}$

\section{Management Algorithm}

A management algorithm is proposed where the first step identifies fracture stability. ${ }^{34}$ If deemed stable, nonoperative management is recommended. A splint that keeps the MCP joint flexed at $90^{\circ}$ and the IP joints in extension is recommended. Occasional active PIP motion may be encouraged as it further compresses and stabilizes the fracture.

It must be stated that a finger undergoing any type of surgery is likely to be stiffer than one that was treated nonoperatively. ${ }^{17,29}$ For that reason, if a fracture is deemed stable yet perhaps not quite enough to begin early active range of motion, then it is perfectly reasonable to delay motion for an additional week or two. A fracture that did not undergo surgery and perhaps progressed more slowly to allow for adequate fracture healing is still better than one that underwent surgery followed by an early range of motion protocol. In this case, all surgical risks are avoided with nonoperative management.

\section{Fracture Location Influences Treatment}

If the fracture is unstable, then fixation in a manner that ensures as much early motion as possible is advised. Regardless of location, this is accomplished by leaving the extensor mechanism as intact as possible and minimizing dissection of the soft tissue envelope.

\section{Proximal Third}

Fractures within the proximal third of the proximal phalanx are approached differently than fractures of the middle or distal thirds. In the proximal third, the lateral bands cloak the proximal phalanx so much so that retained hardware in these locations invariably results in friction with the extensor mechanism, limiting achievement of full range of motion. For that reason, $\mathrm{K}$-wire fixation is particularly advantageous in proximal third fractures, given that this fixation is temporary and is removed once adequate fracture healing has occurred. ${ }^{11}$

Transarticular pins rigidly immobilize the MCP joint and may lead to greater flexor and extensor adhesions when compared with periarticular placement. These pins can be placed after closed or open reduction efforts. When an open reduction is needed, the principle of minimizing extensor mechanism dissection by working on radial and ulnar sides prevails. K-wires can be passed retrograde through the intramedullary canal and drilled through the bone radially and ulnarly. The fracture is then reduced under direct visualization, and the pins are passed antegrade to cross the

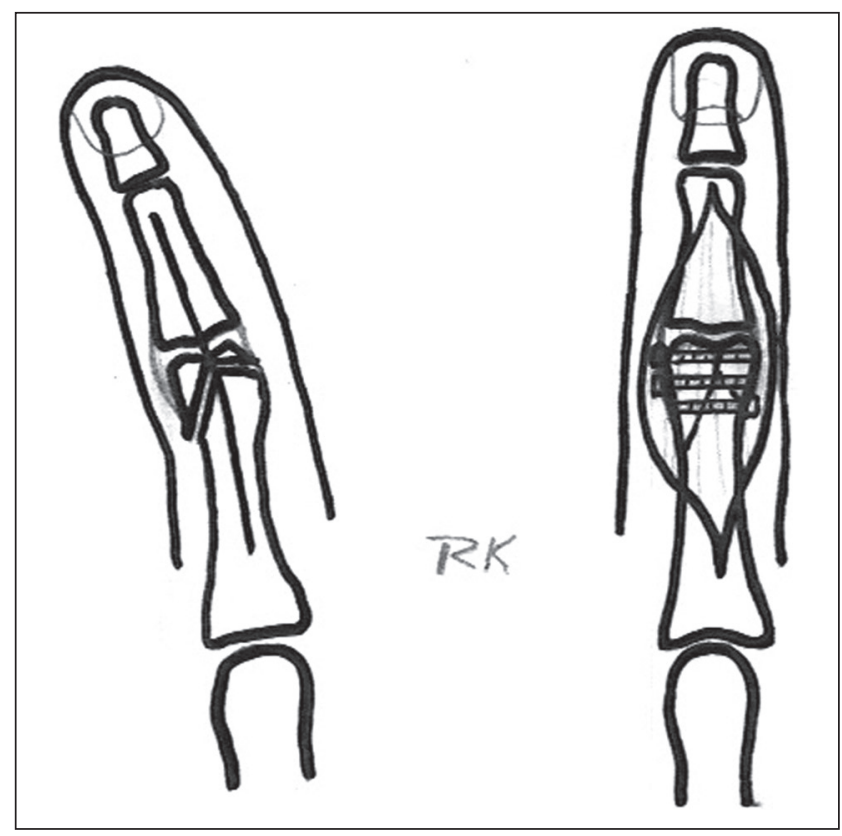

Figure 4. Distal third proximal phalanx fracture with intraarticular extension requires reduction efforts with internal fixation, such as screws.

fracture. The pins are left projecting percutaneously so that they can be removed at 4 weeks. The finger should begin active PIP motion at 3 to 5 days after surgery so that adhesions do not form.

\section{Middle Third}

Middle third fractures benefit from open reduction and internal fixation efforts with plates placed on the radial and ulnar sides where they do not interfere with the gliding of the extensor tendon. Locking screw technology is recommended when the healing environment is particularly compromised, such as in open fractures.

Lag screws are of value and should be countersunk particularly if the screw head projects dorsally. The use of intramedullary screws causes substantial cartilage damage to the joints where the screw enters. The long-term consequences of this are not fully known. Intramedullary screw salvage or removal is predictably difficult given their inaccessibility. Their use cannot be fully recommended at this time given these concerns.

\section{Distal Third}

Distal third fractures are often intra-articular and benefit from open reduction efforts that minimize dissection around the PIP joint (Figure 4). Indirect fracture reduction efforts using provisional $\mathrm{K}$-wires as joysticks to help reduce the fracture are of great value in these locations. Leaving the collateral ligaments attached to the fracture fragment is of great importance. 


\section{Conclusions}

The forces that are transmitted to fingers during early range of motion protocols lie well below those that would cause plate, K-wire, or screw fixation failure. All methods of fixation are permissible as long as adequate stability allows for early postoperative mobilization.

New locking plates and intramedullary screws have been developed to improve phalanx fracture fixation, yet no benefit of these methods has been shown when compared with traditional K-wires, conventional plating, or lag screw fixation methods. Locking screw technology may be advantageous in those fractures that are very unstable and where the healing environment is compromised.

Clinical success is achieved when acceptable fracture alignment and stability encounter unobstructed tendon gliding and early active range of motion.

\section{Ethical Approval}

This study was approved by our institutional review board.

\section{Statement of Human and Animal Rights}

No experiments on animals were performed for this study. No experimental procedures were performed in any human participant for this study.

\section{Statement of Informed Consent}

No informed consent was needed to carry out this study as no individual participants were investigated.

\section{Declaration of Conflicting Interests}

The author(s) declared no potential conflicts of interest with respect to the research, authorship, and/or publication of this article.

\section{Funding}

The author(s) received no financial support for the research, authorship, and/or publication of this article.

\section{References}

1. Al-Qattan MM. Displaced unstable transverse fractures of the shaft of the proximal phalanx of the fingers in industrial workers: reduction and $\mathrm{K}$-wire fixation leaving the metacarpophalangeal and proximal interphalangeal joints free. J Hand Surg Eur Vol. 2011;36(7):577-583.

2. Basar H, Basar B, Basci O, et al. Comparison of treatment of oblique and spiral metacarpal and phalangeal fractures with mini plate plus screw or screw only. Arch Orthop Trauma Surg. 2015;135(4):499-504.

3. Belsky MR, Eaton RG, Lane LB. Closed reduction and internal fixation of proximal phalangeal fractures. J Hand Surg Am. 1984;9(5):725-729.

4. Black DM, Mann RJ, Constine RM, et al. The stability of internal fixation in the proximal phalanx. J Hand Surg Am. 1986;11(5):672-677.
5. Blaine TA, Rosier RN, Puzas JE, et al. Increased levels of tumor necrosis factor-alpha and interleukin- 6 protein and messenger RNA in human peripheral blood monocytes due to titanium particles. J Bone Joint Surg Am. 1996;78(8):1181-1192.

6. Borbas P, Dreu M, Poggetti A, et al. Treatment of proximal phalangeal fractures with an antegrade intramedullary screw: a cadaver study. J Hand Surg Eur Vol. 2016;41(7):683-687.

7. Brei-Thoma P, Vogelin E, Franz T. Plate fixation of extra-articular fractures of the proximal phalanx: do new implants cause less problems? Arch Orthop Trauma Surg. 2015;135(3):439-445.

8. Day CS, Stern PJ. Fractures of the metacarpals and the phalanges. In: Wolfe S, Pederson W, Hotchkiss R, Kozin S, eds. Green's Operative Hand Surgery. Vol. 1. Philadelphia, PA: Elsevier/Churchill Livingstone; 2011:239-290.

9. del Pinal F, Moraleda E, Ruas JS, et al. Minimally invasive fixation of fractures of the phalanges and metacarpals with intramedullary cannulated headless compression screws. J Hand Surg Am. 2015;40(4):692-700.

10. Eberlin KR, Babushkina A, Neira JR, et al. Outcomes of closed reduction and periarticular pinning of base and shaft fractures of the proximal phalanx. J Hand Surg Am. 2014;39(8):15241528.

11. Faruqui S, Stern PJ, Kiefhaber TR. Percutaneous pinning of fractures in the proximal third of the proximal phalanx: complications and outcomes. J Hand Surg Am. 2012;37(7):1342-1348.

12. Figl M, Weninger $\mathrm{P}$, Hofbauer $\mathrm{M}$, et al. Results of dynamic treatment of fractures of the proximal phalanx of the hand. $J$ Trauma. 2011;70(4):852-856.

13. Fowler NK, Nicol AC. Measurement of external three-dimensional interphalangeal loads applied during activities of daily living. Clin Biomech (Bristol, Avon). 1999;14(9):646-652.

14. Franz T, von Wartburg U, Schibli-Beer S, et al. Extraarticular fractures of the proximal phalanges of the fingers: a comparison of 2 methods of functional, conservative treatment. J Hand Surg Am. 2012;37(5):889-898.

15. Giesen T, Gazzola R, Poggetti A, et al. Intramedullary headless screw fixation for fractures of the proximal and middle phalanges in the digits of the hand: a review of 31 consecutive fractures. J Hand Surg Eur Vol. 2016;41(7):688-694.

16. Haynes DR, Rogers SD, Hay S, et al. The differences in toxicity and release of bone-resorbing mediators induced by titanium and cobalt-chromium-alloy wear particles. J Bone Joint Surg Am. 1993;75(6):825-834.

17. Held M, Jordaan P, Laubscher M, et al. Conservative treatment of fractures of the proximal phalanx: an option even for unstable fracture patterns. Hand Surg. 2013;18(2):229-234.

18. Henry M. Soft tissue sleeve approach to open reduction and internal fixation of proximal phalangeal fractures. Tech Hand Up Extrem Surg. 2008;12(3):161-165.

19. Hornbach EE, Cohen MS. Closed reduction and percutaneous pinning of fractures of the proximal phalanx. J Hand Surg Br. 2001;26(1):45-49.

20. Horton TC, Hatton M, Davis TR. A prospective randomized controlled study of fixation of long oblique and spiral shaft fractures of the proximal phalanx: closed reduction and percutaneous Kirschner wiring versus open reduction and lag screw fixation. J Hand Surg Br. 2003;28(1):5-9.

21. Kappos EA, Esenwein P, Meoli M, et al. Implantation of a denaturated cellulose adhesion barrier after plate osteosynthesis of 
finger proximal phalangeal fractures: results of a randomized controlled trial. J Hand Surg Eur Vol. 2016;41(4):413-420.

22. Kozin SH, Thoder JJ, Lieberman G. Operative treatment of metacarpal and phalangeal shaft fractures. J Am Acad Orthop Surg. 2000;8(2):111-121.

23. Kurzen P, Fusetti C, Bonaccio $\mathrm{M}$, et al. Complications after plate fixation of phalangeal fractures. J Trauma. 2006;60(4):841-843.

24. Lins RE, Myers BS, Spinner RJ, et al. A comparative mechanical analysis of plate fixation in a proximal phalangeal fracture model. J Hand Surg Am. 1996;21(6):1059-1064.

25. Nazzal A, Lozano-Calderon S, Jupiter JB, et al. A histologic analysis of the effects of stainless steel and titanium implants adjacent to tendons: an experimental rabbit study. $J$ Hand Surg Am. 2006;31(7):1123-1130.

26. Onishi T, Omokawa S, Shimizu T, et al. Predictors of postoperative finger stiffness in unstable proximal phalangeal fractures. Plast Reconstr Surg. 2015;3(6):e431.

27. Ouellette EA, Dennis JJ, Latta LL, et al. The role of soft tissues in plate fixation of proximal phalanx fractures. Clin Orthop Relat Res. 2004;418:213-218.

28. Page SM, Stern PJ. Complications and range of motion following plate fixation of metacarpal and phalangeal fractures. J Hand Surg Am. 1998;23(5):827-832.
29. Rajesh G, Ip WY, Chow SP, et al. Dynamic treatment for proximal phalangeal fracture of the hand. J Orthop Surg (Hong Kong). 2007;15(2):211-215.

30. Robinson LP, Gaspar MP, Strohl AB, et al. Dorsal versus lateral plate fixation of finger proximal phalangeal fractures: a retrospective study. Arch Orthop Trauma Surg. 2017;137(4):567-572.

31. Rüedi TP, Buckley RE, Moran CG. AO Principles of Fracture Management. New York, NY: Thieme; 2008.

32. Sela Y, Peterson C, Baratz ME. Tethering the extensor apparatus limits PIP flexion following K-wire placement for pinning extra-articular fractures at the base of the proximal phalanx. Hand (N Y). 2016;11(4):433-437.

33. Shimizu T, Omokawa S, Akahane M, et al. Predictors of the postoperative range of finger motion for comminuted periarticular metacarpal and phalangeal fractures treated with a titanium plate. Injury. 2012;43(6):940-945.

34. Windolf J, Siebert H, Werber KD, et al. [Treatment of phalangeal fractures: recommendations of the Hand Surgery Group of the German Trauma Society]. Unfallchirurg. 2008;111(5):331-338; quiz 339.

35. Zelken JA, Hayes AG, Parks BG, et al. Two versus 3 lag screws for fixation of long oblique proximal phalanx fractures of the fingers: a cadaver study. J Hand Surg Am. 2015;40(6):1124-1129. 